\title{
Dust content solutions for the Alcubierre warp drive spacetime
}

\author{
Osvaldo L. Santos-Pereira ${ }^{1, a}$, Everton M. C. Abreu ${ }^{2,3,4, b}$, Marcelo B. Ribeiro ${ }^{1,4,5, c}$ \\ ${ }^{1}$ Instituto de Física, Universidade Federal do Rio de Janeiro-UFRJ, Rio de Janeiro, RJ 21941-972, Brazil \\ 2 Departamento de Física, Universidade Federal Rural do Rio de Janeiro-UFRRJ, Seropédica, RJ 23890-971, Brazil \\ ${ }^{3}$ Departamento de Física, Universidade Federal de Juiz de Fora-UFJF, Juiz de Fora, MG 36036-330, Brazil \\ ${ }^{4}$ Programa de Pós-Graduação Interdisciplinar em Física Aplicada, Instituto de Física, Universidade Federal do Rio de Janeiro-UFRJ, Rio de Janeiro, \\ RJ 21941-972, Brazil \\ ${ }^{5}$ Observatório do Valongo, Universidade Federal do Rio de Janeiro-UFRJ, Rio de Janeiro, RJ 20080-090, Brazil
}

Received: 11 June 2020 / Accepted: 14 August 2020 / Published online: 27 August 2020

(C) The Author(s) 2020

\begin{abstract}
The Alcubierre metric is a spacetime geometry where a massive particle inside a spacetime distortion, called warp bubble, is able to travel at velocities arbitrarily higher than the velocity of light, a feature known as the warp drive. This is a consequence of general relativity, which allows for global superluminal velocities but restricts local speeds to subluminal ones as required by special relativity. In this work we solved the Einstein equations for the Alcubierre warp drive spacetime geometry considering the dust matter distribution as source, since the Alcubierre metric was not originally advanced as a solution of the Einstein equations, but as a spacetime geometry proposed without a source gravity field. We found that all Einstein equations solutions of this geometry containing pressureless dust lead to vacuum solutions. We also concluded that these solutions connect the Alcubierre metric to the Burgers equation, which describes shock waves moving through an inviscid fluid. Our results also indicated that these shock waves behave as plane waves.
\end{abstract}

\section{Introduction}

In general relativity it is possible for particles, in a global sense, to travel with superluminal velocities whereas the light speed limit is respected inside a local light cone. The Alcubierre warp drive metric [1] satisfies this requirement by basically producing a spacetime distortion, called a warp bubble, such that a particle would travel inside this bubble contracting the spacetime in front of it and expanding the spacetime behind it. In such a geometrical arrangement, the particle travels globally with superluminal velocity whereas the warp

\footnotetext{
a e-mail: osvald23@gmail.com

b e-mail: evertonabreu@ufrrj.br

c e-mail: mbr@if.ufrj.br (corresponding author)
}

bubble guarantees that locally the particle's speed remains subluminal. In its original formulation it was advanced that this warp metric would imply the violation of energy conditions, as well as reportedly requiring great amounts of negative energy density.

Following Alcubierre's original work, several efforts were made to understand the main caveats of the warp drive metric. Ford and Roman [2] advanced some quantum inequalities and concluded that large amounts of negative energy would be required to transport particles with small masses across small distances. Hence, these authors concluded that prohibitive huge amounts of negative energy density would be required to create a warp bubble. Using these quantum inequalities, Pfenning and Ford [3] calculated the limits necessary for the bubble parameters and energy values necessary for the viability of the warp drive, concluding then that the energy required for a warp bubble is ten orders of magnitude greater than the total mass of the entire visible universe, also negative.

Krasnikov [4] discussed the possibility of a massive particle making a round trip between two points in space faster than a photon, by arguing that this is not possible when reasonable assumptions for globally hyperbolic spacetimes are made. He discussed in detail some specific spacetime topologies, assuming that, for some of them, they need tachyons for superluminal travel to occur. He also conjectured the need for a possible preparation of a specific spacetime with some devices along the travel path that would be set up previously to operate when they were needed for the superluminal travel to be possible without tachyons. Such spacetime was named a Krasnikov tube by Ref. [5].

Everett and Roman [5] generalized the metric proposed by Krasnikov by hypothesizing a tube along the path of the particle connecting Earth to a distant star. Inside the tube the spacetime is flat, but the lightcones are opened in such a way 
that they allow for superluminal travel in one direction. One of the problems mentioned in Ref. [5] is that even though the Krasnikov tube does not involve closed timelike curves, it is possible to construct a two way non-overlapping tube system such that it would work as a time machine. They also demonstrated that the Krasnikov tube needs great amounts of negative energy density to function. These authors also used the generalized Krasnikov tube metric to calculate an energymomentum tensor (EMT) which would be positive in some specific regions. Further discussions of the metric proposed by Everett and Roman [5] were made by Lobo and Crawford $[6,7]$, who discussed in detail the metric and EMT derived from it, and addressed if it is possible there exists superluminal travel without the weak energy condition violation. The quantum inequalities, brought about from quantum field theory in Ref. [5], were also discussed.

Further studies on this subject were made by the following authors. van de Broeck [8] showed how a minor modification of the Alcubierre geometry can reduce the total energy required for the warp bubble to distort spacetime. He then presented a modification of the original warp drive metric where the total negative mass would be of the order of a few solar masses. Natario [9] argued that both the expansion and the contraction of space for the Alcubierre warp drive is a matter of choice, and proposed a new version of the warp drive theory with zero expansion, a choice of spherical coordinates, and to use the $x$ axis as the polar axis. Lobo and Visser [10] argued that, for the Alcubierre warp drive and its version proposed by Natario [9], the center of the bubble must be massless. They introduced a linearized theory for both approaches and found that even for low velocities the negative energy stored in the warp fields must be just a significant fraction of the particle's mass at the center of the warp bubble. White [11,12] described how a warp field interferometer could be implemented at the Advanced Propulsion Physics Laboratory with the help of the original Alcubierre's ideas [1].

In this paper we investigate some of these issues. Since the Alcubierre metric was not originally advanced as a solution of the Einstein equations, but as an ad hoc proposal aimed at allowing for superluminal global speeds for particles, our aim here is to investigate if the dust energy-momentum tensor, the simplest source matter distribution for the Einstein equations, is able to create a superluminal warp field. We discuss in detail the dust matter distribution together with the Alcubierre warp drive metric. For this matter source the solutions of the Einstein equations require a zero matter density, i.e., vacuum. Nevertheless, the resulting vacuum solutions connect the warp drive metric to the Burgers equation and inviscid fluid with shockwaves, in fact plane waves in the vacuum.

The plan of the paper is as follows. Section 2 briefly reviews the Alcubierre warp drive theory, and in Sect. 3 the non-zero components of the Einstein tensor for the Alcubierre warp drive are written and the energy conditions are discussed. Section 4 presents the Einstein equations written in terms of the dust EMT, analyzes the expressions obtained for the warp drive metric and presents the results. Section 5 depicts our conclusions and final remarks. Appendix I contains a brief description of the Burgers equation.

\section{Warp drive geometry}

This section reviews the main aspects of the Alcubierre warp drive spacetime. The geometrical details of its shape function, which designs the form of the bubble, are presented, and so are its energy conditions.

\subsection{The Alcubierre warp drive spacetime}

The warp drive geometry [1] is basically a spacetime based propulsion system that, in theory, allows a mass particle to travel with apparent velocities greater than the light speed by means of a local spacetime distortion that embeds the particle. The general metric for the warp drive $3+1$ formalism [13] is given by

$$
\begin{aligned}
\mathrm{d} s^{2} & =-\mathrm{d} \tau^{2}=g_{\mu \nu} \mathrm{d} x^{\mu} \mathrm{d} x^{\nu}, \\
& =-\left(\alpha^{2}-\beta_{i} \beta^{i}\right) \mathrm{d} t^{2}+2 \beta_{i} \mathrm{~d} x^{i} \mathrm{~d} t+\gamma_{i j} \mathrm{~d} x^{i} \mathrm{~d} x^{j},
\end{aligned}
$$

where $\mathrm{d} \tau$ is the lapse of proper time, $\alpha$ is the lapse function, $\beta^{i}$ is the spacelike shift vector and $\gamma_{i j}$ is the spatial metric for the hypersurfaces. ${ }^{1}$ The lapse function $\alpha$ and the shift vector $\beta^{i}$ are functions to be determined, whereas $\gamma_{i j}$ is a positivedefinite metric on each of the spacelike hypersurfaces, for all values of time, a feature that makes the spacetime globally hyperbolic. The lapse of proper time $d \tau$ between two adjacent hypersurfaces, measured by those observers moving along the normal direction to the hypersurfaces, also known as Eulerian observers, is described by the following expression:

$\mathrm{d} \tau=\alpha\left(t, x^{i}\right) \mathrm{d} t$.

Figure 1 illustrates the spacetime foliation with two spacelike hypersurfaces $\Sigma_{t}$ and $\Sigma_{t+\mathrm{d} t}$ separated by a timelike distance $\alpha \mathrm{d} t$.

Now, considering the metric (2.1), Alcubierre [1] assumed the following ad hoc particular choices for the parameters,

$$
\alpha=1,
$$

\footnotetext{
${ }^{1}$ From now on Greek indices will range from 0 to 3, whereas Latin ones indicate the spacelike hypersurfaces and will range from 1 to 3 .
} 


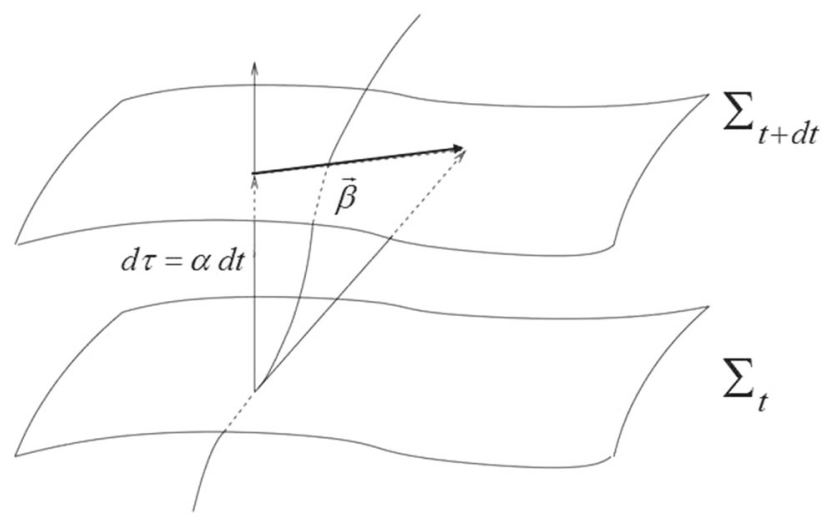

Fig. 1 Two adjacent spacelike hypersurfaces showing the definitions of the lapse function $\alpha$ and the shift vector $\beta^{i}$. It is clear that the shift vector $\boldsymbol{\beta}$ represents how the spacelike coordinates change from one hypersurface to another as the proper time elapses. For more details about this $3+1$ formalism the reader is refereed to Ref. [13, Chaps. 1-2] for a clear and concise explanation

$$
\begin{aligned}
& \beta^{1}=-v_{s}(t) f\left[r_{s}(t)\right], \\
& \beta^{2}=\beta^{3}=0, \\
& \gamma_{i j}=\delta_{i j} .
\end{aligned}
$$

Hence, Eq. (2.1) becomes what may be called the Alcubierre warp drive metric. It may be written

$$
\begin{aligned}
\mathrm{d} s^{2}= & -\left[1-v_{s}(t)^{2} f\left(r_{s}\right)^{2}\right] \mathrm{d} t^{2}-2 v_{s}(t) f\left(r_{s}\right) \mathrm{d} x \mathrm{~d} t+\mathrm{d} x^{2} \\
& +\mathrm{d} y^{2}+\mathrm{d} z^{2},
\end{aligned}
$$

where $v_{s}(t)$ is the velocity of the center of the bubble moving along the curve $x_{s}(t)$. This is given by the following expression:

$v_{s}(t)=\frac{\mathrm{d} x_{s}(t)}{\mathrm{d} t}$.

The function $f\left(r_{s}\right)$, named by Alcubierre [1] as the warp metric regulating function, describes the shape of the warp bubble. The interior of the bubble is an inertial reference frame and the observers within it suffer no proper acceleration. A photon within the warp bubble would always move faster than a mass particle, as it should according to special relativity. The regulating function $f\left(r_{s}\right)$ is defined as follows:

$f\left(r_{s}\right)=\frac{\tanh \left[\sigma\left(r_{s}+R\right)\right]-\tanh \left[\sigma\left(r_{s}-R\right)\right]}{2 \tanh (\sigma R)}$,

being, therefore, determined by two arbitrary and positive parameters, $\sigma$ and $R$. The former is inversely related to the thickness of the warp bubble, and the latter is proportional to the bubble's radius. The variable $r_{s}(t)$ is defined as the distance from the center of the bubble $\left[x_{s}(t), 0,0\right]$ to a generic point $(x, y, z)$ on the surface of the bubble, as the following expression shows:

$r_{S}(t)=\sqrt{\left[x-x_{S}(t)\right]^{2}+y^{2}+z^{2}}$.
If one substitutes $x=x_{s}(t)$ in the warp drive metric in Eq. (2.7) it is straightforward to show that a particle inside the bubble moves on a geodesic (see Ref. [1]) regardless of the value of $v_{s}(t)$. Hence, the geodesic $x=x_{S}(t)$ is physically interpreted as the mass particle trajectory having no time dilatation, as can be readily seen by the fact that $d \tau=\alpha d t$, and by the choice of $\alpha=1$.

The regulating function can be approximated by a step function, because for distances where $\left|r_{s}(t)\right|<R$ then $f\left(r_{s}\right)=1$, whereas for distances where $\left|r_{s}(t)\right| \gg R$ then $f\left(r_{s}\right) \rightarrow 0$. Hence,

$\lim _{\sigma \rightarrow \infty} f\left(r_{s}\right)= \begin{cases}1, & \text { for } r_{s} \in[-R, R] \\ 0, & \text { otherwise. }\end{cases}$

Note that when the regulating function is equal to zero, that is, outside the warp bubble, according to Eq. (2.7) the spacetime is flat.

\subsection{Caveats and main points}

Ref. [1] emphasized some fundamental concepts of special and general relativity theories by arguing that the main theoretical point behind the warp drive concept is that general relativity (GR) does not forbid superluminal velocities in a global sense because spacetime is dynamic. In the $3+1$ formalism the foliation of hyperspace with a time parameter is a perfect tool to allow a clear interpretation of the results and to prevent the use of closed causal curves. The warp drive metric relies on the regulating function $f\left(r_{s}\right)$ to describe the form of the warp bubble.

A simple thought experiment can be advanced in order to demonstrate that it is possible for particles to make round trips with superluminal velocity. A particle moving in a local light cone can make a round trip between points A and B separated by a distance $D$ in a time less than $2 D / c$ measured by an observer that remains always at the place of departure by just using contraction and expansion of spacetime. Alcubierre also stated [1] that the warp drive is possible without the use of non-trivial topologies, such as wormholes. The particle trajectories are always a timelike curve, regardless of the parameters used in the Alcubierre metric. The proper time of distant observers in a flat region is equal to the coordinate time. This implies that the particle suffers no time dilatation as it moves on a geodesic.

One of the major issues that seemed to imply the unphysical nature of superluminal velocities by means of the Alcubierre geometry came from the initial perception that the warp drive metric violates the three energy conditions: weak, dominant and strong. By using Eulerian observers and the warp drive metric the calculations showed that the energy density becomes everywhere negative [1], a result that implies the violation of both the weak and the domi- 
nant energy conditions. However, this result did not come from the formal solutions of the Einstein equations, but by finding the Einstein tensor for the warp drive metric and contracting it to the 4-velocity. By applying the weak energy condition Ref. [1] showed that the energy density must be negative. In other words, similar to wormholes, superluminal speeds would require exotic matter. However, such a requirement does not necessarily eliminate the possibility of using a spacetime distortion mechanism for achieving a warp drive propulsion system, that is, a hyper-fast interstellar travel, because Ref. [1] claimed that although exotic matter may be forbidden classically, quantum field theory permits the existence of regions with negative energy densities, this being the case of the Casimir effect [14].

Finally, spacetime topology means that the spacetime described by the Alcubierre metric is globally hyperbolic. It is then possible to construct a spacetime that contains closed causal curves using an idea similar to the one used in Ref. [1].

\section{The Einstein tensor}

As mentioned above, the Alcubierre metric was not proposed as a solution of the Einstein field equations, but simply as a metric whose properties are equivalent to a propulsion system that drives a mass particle at superluminal global speeds by "warping" the spacetime, that is, by generating warp speeds. Hence, the question that may be posed is what kind of matter or field sources would be able to produce such spacetime properties. To follow this analytical path one should couple the Alcubierre metric to the Einstein field equations in order to try to solve the resulting equations and draw some conclusions.

\subsection{Einstein tensor components}

We shall start by adopting Alcubierre's original notation of $\beta=-\beta^{1}=v_{s}(t) f\left(r_{s}\right)$ in Eq. (2.4), since the other shift vectors are zero. The components of the Einstein tensor without a cosmological constant for the warp drive metric (2.7) are given by the expressions

$$
\begin{aligned}
G_{00}= & -\frac{1}{4}\left(1+3 \beta^{2}\right)\left[\left(\frac{\partial \beta}{\partial y}\right)^{2}+\left(\frac{\partial \beta}{\partial z}\right)^{2}\right] \\
& -\beta\left(\frac{\partial^{2} \beta}{\partial y^{2}}+\frac{\partial^{2} \beta}{\partial z^{2}}\right), \\
G_{01}= & \frac{3}{4} \beta\left[\left(\frac{\partial \beta}{\partial y}\right)^{2}+\left(\frac{\partial \beta}{\partial z}\right)^{2}\right]+\frac{1}{2}\left(\frac{\partial^{2} \beta}{\partial y^{2}}+\frac{\partial^{2} \beta}{\partial z^{2}}\right),
\end{aligned}
$$

$$
\begin{aligned}
G_{02}= & -\frac{1}{2} \frac{\partial^{2} \beta}{\partial x \partial y}-\frac{\beta}{2}\left(2 \frac{\partial \beta}{\partial y} \frac{\partial \beta}{\partial x}+\beta \frac{\partial^{2} \beta}{\partial x \partial y}+\frac{\partial^{2} \beta}{\partial t \partial y}\right) \\
G_{03}= & -\frac{1}{2} \frac{\partial^{2} \beta}{\partial x \partial z}-\frac{\beta}{2}\left(2 \frac{\partial \beta}{\partial z} \frac{\partial \beta}{\partial x}+\beta \frac{\partial^{2} \beta}{\partial x \partial z}+\frac{\partial^{2} \beta}{\partial t \partial z}\right) \\
G_{11}= & -\frac{3}{4}\left[\left(\frac{\partial \beta}{\partial y}\right)^{2}+\left(\frac{\partial \beta}{\partial z}\right)^{2}\right] \\
G_{12}= & \frac{1}{2}\left(2 \frac{\partial \beta}{\partial y} \frac{\partial \beta}{\partial x}+\beta \frac{\partial^{2} \beta}{\partial x \partial y}+\frac{\partial^{2} \beta}{\partial t \partial y}\right) \\
G_{13}= & \frac{1}{2}\left(2 \frac{\partial \beta}{\partial z} \frac{\partial \beta}{\partial x}+\beta \frac{\partial^{2} \beta}{\partial x \partial z}+\frac{\partial^{2} \beta}{\partial t \partial z}\right) \\
G_{23}= & \frac{1}{2} \frac{\partial \beta}{\partial z} \frac{\partial \beta}{\partial y}, \\
G_{22}= & -\left[\frac{\partial^{2} \beta}{\partial t \partial x}+\beta \frac{\partial^{2} \beta}{\partial x^{2}}+\left(\frac{\partial \beta}{\partial x}\right)^{2}\right] \\
& -\frac{1}{4}\left[\left(\frac{\partial \beta}{\partial y}\right)^{2}-\left(\frac{\partial \beta}{\partial z}\right)^{2}\right], \\
& +\frac{1}{4}\left[\left(\frac{\partial \beta}{\partial y}\right)^{2}-\left(\frac{\partial \beta}{\partial z}\right)^{2}\right] . \\
G_{33}= & -\left[\frac{\partial^{2} \beta}{\partial t \partial x}+\beta \frac{\partial^{2} \beta}{\partial x^{2}}+\left(\frac{\partial \beta}{\partial x}\right)^{2}\right] \\
& 13
\end{aligned}
$$

\subsection{Energy conditions}

The components for the Eulerian (normal) observers' 4velocities are given by

$u^{\alpha}=\left[1,-v_{s}(t) f\left(r_{s}\right), 0,0\right], \quad u_{\alpha}=(-1,0,0,0)$.

Using, as below, these results in the Einstein equations,

$T_{\alpha \beta} u^{\alpha} u^{\beta}=\frac{1}{8 \pi} G_{\alpha \beta} u^{\alpha} u^{\beta}$,

allows us to obtain an expression concerning the energy conditions. Considering Eq. (3.11) and that the only non-zero terms of Eq. (3.12) are $G_{00}, G_{01}$ and $G_{11}$, we obtain the following expression:

$T_{\alpha \beta} u^{\alpha} u^{\beta}=\frac{1}{8 \pi}\left(G_{00}-2 v_{s} f G_{01}+v_{s}^{2} f^{2} G_{11}\right)$.

Substituting Eqs.(3.1), (3.2) and (3.5) into Eq. (3.13) the result may be written

$T_{\alpha \beta} u^{\alpha} u^{\beta}=-\frac{v_{s}^{2}}{32 \pi}\left[\left(\frac{\partial f}{\partial y}\right)^{2}+\left(\frac{\partial f}{\partial z}\right)^{2}\right]$.

This expression can be physically interpreted as being a matter-energy density as observed in the frame of Eulerian 
observers. Besides, since the bubble radius is given by

$r_{s}=\sqrt{\left(x-x_{s}\right)^{2}+y^{2}+z^{2}}$,

applying implicit partial derivative rules, Eq. (3.14) takes then the form below,

$T_{\alpha \beta} u^{\alpha} u^{\beta}=-\frac{v_{s}^{2}}{16 \pi} \frac{y^{2}+z^{2}}{r_{s}^{2}}\left(\frac{\partial f}{\partial r_{s}}\right)^{2} \leq 0$.

The expression above shows that the energy density can only vanish or assume negative values, a result that Ref. [1] assumed as being a necessary condition for exotic matter and faster than light travel. In addition, Ref. [1] also emphasized that this result violates the energy conditions because the energy density becomes everywhere negative. There are four energy conditions in general relativity [15] and, according to Alcubierre, the warp drive metric violates both the weak and the dominant ones.

Nevertheless, it must be noted that although Alcubierre stated [1, Eq. 19] that the relation $T_{\alpha \beta} u^{\alpha} u^{\beta}$ must be everywhere negative, the weak, strong, null, and dominant energy conditions can still be satisfied if this contraction is equal to zero [15].

\section{Dust content energy-momentum tensors}

In this section we shall discuss matter content solutions of the Einstein's equations considering the dust EMT for the Alcubierre metric. This is the simplest possible matter content that can be studied as possible source for warp speeds. The dust solution contains only matter-energy density and depends on the 4-velocities of the observables $T_{\alpha \beta}=\mu u_{\alpha} u_{\beta}$. From now on we shall assume the following form for the Einstein field equations:

$G_{\mu \nu}=8 \pi T_{\mu \nu}$

\subsection{Dust warp metric solutions}

The dust solution is an exact solution of the Einstein equations for fluids where gravity is produced by the mass density of pressureless particles. It can be understood as a model for a configuration of dust particles that move with gravity alone and, hence, there is no other type of interaction among them. This solution is used to model gravitational collapse, besides use in in cosmology, since galaxies are considered the basic building blocks of the universe whose main interaction is due to the general geometrical background. One can further envisage a possible interest in this solution if one considers the galactic disks as being modeled by finite rotating disks of dust.

The stress-energy tensor of a relativistic fluid with no pressure can be written in the simple form
$T_{\alpha \beta}=\mu u_{\alpha} u_{\beta}$

where $\mu$ is a scalar function that represents the matter density. Considering Eq. (3.11), the stress-energy tensor yields

$T_{\alpha \beta}=\left(\begin{array}{cccc}\mu & 0 & 0 & 0 \\ 0 & 0 & 0 & 0 \\ 0 & 0 & 0 & 0 \\ 0 & 0 & 0 & 0\end{array}\right)$.

In order to solve the Einstein equations one has to use the tensor components given in Eqs. (3.1) to (3.10) with the dust EMT above. Substituting $G_{11}=8 \pi T_{11}$ and $G_{01}=$ $8 \pi T_{01}$ from the Einstein equations into the component $G_{00}=$ $8 \pi T_{00}$, the resulting expression may be written as follows:

$T_{00}+2 \beta T_{01}+\frac{1}{3}\left(3 \beta^{2}-1\right) T_{11}=0$.

Now, considering Eq. (4.3) in the equation above implies, in a vanishing matter density,

$\mu=0$.

This result implies that a warp bubble cannot be created with a dust matter distribution as source. Nevertheless, the other components of the Einstein equations lead to some interesting features for the Alcubierre warp drive metric, as we shall see below.

Since $T_{23}=0$, the result below follows from the equation $G_{23}=8 \pi T_{23}$,

$G_{23}=\frac{1}{2} \frac{\partial \beta}{\partial z} \frac{\partial \beta}{\partial y}=0$,

which means that either $\partial \beta / \partial z$, or $\partial \beta / \partial y$, or both, vanish. Let us now discuss both cases.

Case 1: $\left[\frac{\partial \boldsymbol{\beta}}{\partial z}=\mathbf{0}\right]$ This means that the function $\beta$ does not depend on the coordinate $z$ and the Einstein tensor components $G_{13}, G_{03}$ and $G_{23}$ are identically zero. Substituting $\partial \beta / \partial z=0$ into the component $G_{11}=8 \pi T_{11}$, and since $T_{11}=0$, it follows immediately that

$\frac{\partial \beta}{\partial y}=0$

which means that the function $\beta$ does not depend on the $y$-coordinate either. From Eq. (4.3) it is straightforward to verify that the components $G_{22}=8 \pi T_{22}$, and $G_{33}=$ $8 \pi T_{33}$ are also zero. Therefore, the field equations are reduced to

$\mu=0$,

$\frac{\partial^{2} \beta}{\partial t \partial x}+\beta \frac{\partial^{2} \beta}{\partial x^{2}}+\left(\frac{\partial \beta}{\partial x}\right)^{2}=0$. 
Case 2: $\left[\frac{\partial \beta}{\partial y}=0\right]$ This means that the function $\beta$ does not depend on the $y$-coordinate and, consequently, the Einstein tensor components $G_{12}, G_{23}$ and $G_{02}$ are identically zero. Since $G_{11}=8 \pi T_{11}$, and $T_{11}=0$, it follows immediately that

$$
\frac{\partial \beta}{\partial z}=0,
$$

which means that the function $\beta$ does not depend on the $z$-coordinate either. Hence, the set of field equations are also reduced to the expressions (4.8) and (4.9).

The two cases above lead to the same results, so it does not matter if $\partial \beta / \partial y=0$ or $\partial \beta / \partial z=0$. In addition, they both lead to a vanishing matter density $\mu=0$ and, consequently, the energy density found in Eqs. (3.14) and (3.16) must be zero. This means that the energy conditions in Eqs. (3.16) are immediately, and trivially, satisfied. One must mention that such a trivial result is a consequence of the dust case EMT in the Alcubierre warp drive metric leading back to a vacuum solution, which needs not necessarily happen when one considers more complex dust or energy content EMTs.

Nevertheless, one is still left with a single partial differential equation to solve, Eq. (4.9), which can be rewritten

$\frac{\partial}{\partial x}\left[\frac{\partial \beta}{\partial t}+\frac{1}{2} \frac{\partial}{\partial x}\left(\beta^{2}\right)\right]=0$,

whose integration is straightforward if we remember that $\beta=$ $\beta(t, x)$, yielding

$\frac{\partial \beta}{\partial t}+\frac{1}{2} \frac{\partial}{\partial x}\left(\beta^{2}\right)=h(t)$,

where $h=h(t)$ is an arbitrary function of $t$, to be determined by boundary conditions.

In its homogeneous form, that is, for $h(t)=0$, Eq. (4.12) becomes the conservative form of the inviscid Burgers equation (see Appendix I for details), a well-known equation appearing in fluid models, such as gas dynamics and traffic flows, as well as in hyperbolic equations and conservation laws. It is a quasilinear hyperbolic equation and its current density is the kinetic energy density. If one defines the flow density as being given by $J_{f}=J_{f}(\beta)$, which may be a general function of $\beta$, and let it be given by the following expression:

$J_{f}(\beta)=\beta^{2}$,

Eq. (4.12) may be rewritten

$\frac{\partial \beta}{\partial t}+\frac{1}{2} \frac{\partial}{\partial x} J_{f}=h(t)$.

The phenomena arising from the Burgers equation are conservation laws and the formation of shock waves, that is, dis-
Table 1 Summary of results for the warp drive spacetime having dust matter content

\begin{tabular}{lll}
\hline Case & Consequence & Results \\
\hline (1) $\frac{\partial \beta}{\partial z}=0$ & $\frac{\partial \beta}{\partial y}=0$ & $\mu=0$ \\
& & $\beta=\beta(t, x)$ \\
& $\frac{\partial \beta}{\partial t}+\frac{1}{2} \frac{\partial}{\partial x}\left(\beta^{2}\right)=h(t)$ \\
(2) $\frac{\partial \beta}{\partial y}=0$ & $\frac{\partial \beta}{\partial z}=0$ & $\beta=0$ \\
& & $\frac{\partial \beta}{\partial t}+\frac{1}{2} \frac{\partial}{\partial x}\left(\beta^{2}\right)=h(t)$ \\
\hline
\end{tabular}

continuities that appear after a finite time and then propagate in a regular manner. The one dimensional conservation law implicit in Eq. (4.14) can be seen if we note that equations of the form

$\frac{\partial u}{\partial t}+\frac{\partial}{\partial x} F(u)=0$

can be interpreted as a conservation law [16], where the function $u=u(t, x)$ is to be determined with the initial condition

$u(t=0, x)=u_{0}(x)$.

The Burgers equation can describe rarefaction and expansion waves. Hence, in the present context Eq. (4.14) can depict a spacetime shock wave, in this case a plane wave. It is worth noticing that Alcubierre built a warp drive bubble in vacuum, so when we impose the dust solution to the EMT and solve the Einstein equations for the warp drive metric the matter density vanishes, recovering the vacuum, but also showing that the warp bubble regulating function may obey the inviscid Burgers equation in the particular case when the function $h(t)$ vanishes.

In the warp drive scenario, $\beta=v_{s}(t) f\left(r_{s}\right)$ would be interpreted as a boost in the $x$-direction, which means that the warp bubble obeying the more general Burgers equation (4.12) the warp drive may then be understood as conservation of linear momentum in the $x$-direction when $h(t)=$ constant. Then $\partial \beta / \partial t$ may be interpreted as a force per unit mass, i.e., the time derivative of momentum, and $(1 / 2) \partial\left(\beta^{2}\right) / \partial x$ would be a potential, i.e., the divergence of the total energy that is entirely kinetic. This would seem reasonable, since the EMT for the dust solution implies no interaction among particles, so the self-gravitating potential is neglected. Note that both cases 1 and 2 above lead to the same results, being then a consequence of the symmetric properties of both the Einstein equations and the EMTs. Table 1 summarizes the results obtained above.

\subsection{The warp metric and shock waves}

We have seen that the Burgers equation appeared here as a vacuum solution of the warp drive metric when one con- 
siders this geometry in the Einstein equations with dust matter source. In recap, the current density was given by $\beta=v_{s}(t) f\left(r_{s}\right)$, where $v_{s}$ is the bubble velocity, $f\left(r_{s}\right)$ is the regulating function of the bubble shape and the inviscid Burgers equation (4.15) represents a conservation law for this current density. This result can be physically understood as a conservation law. Analyzing each term of the Burgers equation as in Eq. (4.12), the first term in the left hand side, $\partial \beta / \partial t$, can be interpreted as a type of force per unit mass, i.e., the time derivative of momentum, since for the warp drive $\beta=v_{s}(t) f\left(r_{s}\right)$ and this function contains the bubble velocity and shape in the $x$-direction. The second term on the left hand side, $\frac{1}{2} \partial\left(\beta^{2}\right) / \partial x$, can be understood as the divergence of the total energy, which is entirely kinetic. The right hand side is a function $h(t)$ of the time coordinate only, which can be determined by boundary conditions. When $h(t)=0$ the inviscid Burgers equation is recovered, which is a kind of conservation equation. Physically it can be understood considering the warp metric in terms of the conservation of energy and momentum in the direction of the wave propagation.

The dust solutions lead to zero matter density, and the Burgers equation arose in this context as a regulating function parameter. This result suggests that the necessary energy to create the associate shock wave is purely geometrical. The fact that the Burgers equation appeared as part of the solution of the Einstein equations relative to the vacuum solution is very interesting evidence that the warp drive metric can be understood as spacetime motion equivalent to a shock wave moving in a fluid.

As an analogy, shock waves are produced when, for example, an aircraft traveling at high subsonic velocity produces sound waves piling up due to the air surrounding the aircraft traveling at local speed of sound, causing a kind of explosion. This result may be considered intuitive for the perfect fluid, but it is not clear if thisis the case for dust because, as seen above, in this case it happens in vacuum.

\subsection{Divergence for the Dust EMT}

Calculating the divergence for the dust EMT, and requiring that it should be null, one arrives at the following condition:

$\mu \frac{\partial \beta}{\partial x}=0$,

which is immediately satisfied since the matter density vanishes for the dust EMT.

\section{Conclusions}

In this work we have analyzed the solutions of the Einstein equations for the Alcubierre warp drive spacetime with the choice of the dust energy-momentum tensor (EMT) as a pos- sible source of global superluminal particle velocities, that is, warp speeds. The Einstein equations are reduced to two solutions that lead to the same results. The matter density becomes equal to zero, $\beta(t, x)$ becomes a function of the time and $x$ coordinates only and the Burgers equation appears as a special case of the vacuum warp drive spacetime. The divergence for the dust EMT is zero. In addition, all the energy conditions are trivially satisfied.

Summing up, we showed that if one starts with dust only EMT the Alcubierre type warp drive is not possible, since the Einstein equations lead the solutions back to vacuum. A more complex matter distribution source for the EMT than simple dust is possibly required for a warp drive bubble including, perhaps, some eletromagnetic components in the EMT or even including the cosmological constant in the Einstein equations. These issues are the subject of ongoing research.

Regarding Eq. (4.12), two possibilities are worth considering. If $h(t)=0$ the inviscid Burgers equation is recovered, which is a type of conservation equation with discontinuities, that is, shock waves, in the case studied here we have plane waves. If $h(t)$ were to be of the form

$h(t)=v \frac{\partial^{2} \beta}{\partial x^{2}}$,

then Eq. (4.12) becomes the viscous Burgers equation, valid for a dissipative system, where $v$ is a diffusion coefficient. It must also be mentioned that the Burgers equation is found in other relativistic solutions, such as FLRW cosmology and the Schwarzschild background [17-19]. Based on Eq. (5.1), one might speculate that $h(t)$ possibly acts as a source term that generates the shock waves.

Acknowledgements We are grateful to an anonymous referee for important insights, suggestions and recommendations that improved the paper. E.M.C.A. thanks CNPq (Conselho Nacional de Desenvolvimento Científico e Tecnológico), Brazil's government scientific supporting agency, for partial financial support, Grants numbers 406894/2018-3 and 302155/2015-5.

Data Availability Statement This manuscript has no associated data or the data will not be deposited. [Authors' comment: This is a theoretical work. There is no associated data.]

Open Access This article is licensed under a Creative Commons Attribution 4.0 International License, which permits use, sharing, adaptation, distribution and reproduction in any medium or format, as long as you give appropriate credit to the original author(s) and the source, provide a link to the Creative Commons licence, and indicate if changes were made. The images or other third party material in this article are included in the article's Creative Commons licence, unless indicated otherwise in a credit line to the material. If material is not included in the article's Creative Commons licence and your intended use is not permitted by statutory regulation or exceeds the permitted use, you will need to obtain permission directly from the copyright holder. To view a copy of this licence, visit http://creativecomm ons.org/licenses/by/4.0/.

Funded by SCOAP $^{3}$. 


\section{Appendix I: The Burgers equation}

The Burgers equation is a very famous nonlinear partial differential equation due to its application in areas such as fluid and gas dynamics, traffic flow, acoustics, shock waves, and so forth. The equation was first discovered by Forsyth [20] and later by Bateman [21]. However, the equation was named after Burgers [22] because of his extensive work upon the issue. The general form of the viscous Burgers equation in one space dimension is

$\frac{\partial u}{\partial t}+u \frac{\partial u}{\partial x}=v \frac{\partial^{2} u}{\partial x^{2}}$,

where $u(x, t)$ is field dependent as regards both time $t$ and the $x$-coordinate, and $v=v(u, x, t)$ is the diffusion coefficient. In this form it is known from its use in modeling dissipative systems. When the diffusion term is zero, $v=0$, the Burgers equation assumes its inviscid form, which means that the equation is free of the viscosity term,

$\frac{\partial u}{\partial t}+u \frac{\partial u}{\partial x}=0$.

This can also be rewritten in its conservative form:

$\frac{\partial u}{\partial t}+\frac{1}{2} \frac{\partial}{\partial x}\left(u^{2}\right)=0$.

The inviscid form of the Burgers equation can be classified as a quasilinear hyperbolic equation, used as a model for a conservation equation. The solution of the inviscid Burgers equation can be constructed by using the characteristics method, namely, for an initial condition $u(x, 0)=g(x)$, the solution is given by a wave solution of the form $u(x, t)=$ $g(x-u t)$. The interested reader will find in Ref. [16] a discussion about the explicit solution of the inviscid Burgers equation.

The solution $u(x, t)=g(x-u t)$ is written in such a way that its characteristics do not intersect each other. Besides, when they do intersect, the inviscid Burgers equation leads to a shock wave framework, which is considered as a propagation of a perturbation. See Ref. [23] for more details on shock wave theory in viscous fluids and how it relates to turbulence theory.

When one thinks about waves in fluids, the shock waves appear when the waves move faster than the local velocity of sound in this very fluid. They can be characterized by an abrupt modification of the pressure, temperature and density of the medium. Shock wave velocity and energy dissipate quickly as a function of the distance. Another interesting feature concerning shock waves is that they keep the energy, but they increase the entropy of the system [16]. Consequently, the decrease in energy for a shock wave can be transformed into work in order to keep energy for the system.

In the warp drive scenario, when the dust EMT solution was proposed, the current density of the Burgers equation appeared as $v_{s}^{2} f^{2}$. Hence, it can be understood as the conservation of the kinetic energy field of the warp bubble.

\section{References}

1. M. Alcubierre, The warp drive: hyper-fast travel within general relativity. Class. Quant. Grav. 11, L73 (1994). arXiv:gr-qc/0009013

2. L.H. Ford, T.A. Roman, Quantum field theory constrains traversable wormhole geometries. Phys. Rev. D 53, 5496 (1996) arXiv:gr-qc/9510071

3. M.J. Pfenning, L.H. Ford, The unphysical nature of Warp Drive. Class. Quant. Grav. 14, 1743 (1997). arXiv:gr-qc/9702026

4. S.V. Krasnikov, Hyperfast interstellar travel in general relativity. Phys. Rev. D 57, 4760 (1998). arXiv:gr-qc/9511068

5. A. Everett, T.A. Roman, A superluminal subway: the krasnikov tube. Phys. Rev. D 56, 2100 (1997). arXiv:gr-qc/9702049

6. F.S.N. Lobo, P. Crawford, Weak energy condition violation and superluminal travel. Lect. Notes Phys. 617, 277 (2003). arXiv:gr-qc/0204038

7. F.S.N. Lobo, P. Crawford, Weak energy condition violation and superluminal Travel. In: Fernández-Jambrina L., GonzálezRomero L.M. (eds), Current Trends in Relativistic Astrophysics, Springer, Berlin (2003). arXiv:gr-qc/0204038

8. C. Van Den Broeck, A warp drive with more reasonable total energy. Class. Quant. Grav. 16, 3973 (1999). arXiv:gr-qc/9905084

9. J. Natario, Warp drive with zero expansion. Class. Quant. Grav. 19, 1157 (2002). arXiv:gr-qc/0110086

10. F.S.N. Lobo, M. Visser, Linearized warp drive and the energy conditions, (2004). arXiv:gr-qc/0412065

11. H.G. White, A Discussion of Space-Time Metric Engineering. Gen. Relat. Grav. 35, 2025 (2003)

12. H.G. White, Warp field mechanics 101. J. Br. Interplanet. Soc. 66, $242(2011)$

13. M. Alcubierre, Introduction to $3+1$ Numerical Relativity (Oxford University Press, Oxford, 2012)

14. B.S. DeWitt, In General Relativity: An Einstein Centenary Survey, S.W. Hawking and W. Israel (eds), Cambridge University Press, (1980)

15. S.W. Hawking, G.F.R. Ellis, The Large Scale Structure of SpaceTime (Cambridge University Press, Cambridge, 1973)

16. L.C. Evans, Partial differential equations. Grad. Stud. Math. Am. Math. So. 19, 749 (2010)

17. T. Ceylan, B. Okutmuştur, Finite volume approximation of the relativistic Burgers equation on a Schwarzschild (anti-)de Sitter spacetime. Turkish J. Math. 41, 1027 (2017)

18. T. Ceylan, B. Okutmuştur, The relativistic Burgers equation on a de Sitter spacetime. Derivation and finite volume approximation. Int. J. Pure. Math. 2, 20 (2015)

19. P.G. LeFloch, H. Makhlof, B. Okutmuştur, Relativistic Burgers equation on curved spacetime. Derivation and finite volume approximation. arXiv: 1206.3018

20. A.R. Forsyth, Theory of Differential Equations (Cambridge University Press, Cambridge, 1906)

21. H. Bateman, Some recent researches on the motion of fluids. Mon. Weather Rev. 43, 163 (1915)

22. J.M. Burgers, A Mathematical Model illustrating the Theory of Turbulence Advances in Applied Mechanics, vol. 1 (Elsevier, Oxford, 1948), pp. 171-199

23. J.D. Cole, On a quasi-linear parabolic equation occurring in aerodynamics. Q. Appl. Math. 9, 225 (1951) 